\title{
The protective effects of eugenol on metabolic-syndrome, renal damages
}

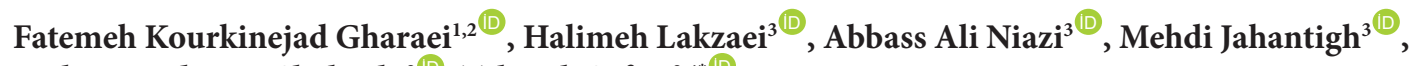 \\ Mohammad Reza Shahraki ${ }^{3 \oplus}$, Tahereh Safari ${ }^{3,4^{*}}$ \\ ${ }^{1}$ Faculty of Medicine, Zahedan University of Medical Sciences, Zahedan, Iran \\ ${ }^{2}$ Student Research Committee, Zahedan University of Medical Sciences, Zahedan, Iran \\ ${ }^{3}$ School of Medicine, Department of Physiology, Zahedan University of Medical Sciences, Zahedan, Iran \\ ${ }^{4}$ Pharmacology Research Center, Zahedan University of Medical Sciences, Zahedan, Iran
}

\section{A R T I C L E I N F O}

\section{Article Type:}

Original

\section{Article History:}

Received: 15 July 2020

Accepted: 23 September 2020

Published online: 8 October 2020

\section{Keywords:}

Eugenol

Metabolic syndrome

Kidney injury

\begin{abstract}
A B S T R A C T
Introduction: Metabolic syndrome consists of a group of abnormities which is involved with chronic kidney disease and nephropathy. Eugenol is an important phenolic component, which is present in many plants' essential oils such as cloves oil with antioxidant effects.

Objectives: Our study planned to demonstrate eugenol's effects over nephrotoxicity derived from metabolic syndrome.

Materials and Methods: Thirty-five male Wistar rats were picked accidentally and then divided into five groups including 1) tap water; 2) water with fructose10\%; 3) water with fructose + sweet almond oil and administered intraperitoneally; 4) water with fructose+ eugenol $50 \mathrm{mg} / \mathrm{kg} / \mathrm{d}$ and administered intraperitoneally; 5) water with fructose+ eugenol 100 $\mathrm{mg} / \mathrm{kg} / \mathrm{d}$ administered intraperitoneally. This regime lasted for 60 days, and at the beginning of day 31st, injections started for 30 days. Assessment of serum, urine and renal parameters (in homogenized kidney tissue) were conducted in the last step.

Results: The results argued that the induction of metabolic syndrome following renal injury has significantly increased serum blood urea nitrogen (BUN) and creatinine (Cr) levels in the fructose group. Consumption of eugenol resulted in a significant reduction in the level of these two biochemical factors $(P<0.05)$. The renal level of malondialdehyde $(\mathrm{MDA})$ increased in the fructose group while treatment with a dose of 50 eugenol decreasing its level $(P<0.05)$. Proteinuria and kidney tissue damage score (KTDS) increased in the fructose group compared with the tap water group $(P<0.001)$. It is noteworthy that treatment with eugenol did not affect the level of proteinuria and KTDS with any of the used doses.

Conclusion: Our results indicated the improvement of renal functioning and decrease in lipid peroxidation, although eugenol doses used in this study did not reduce proteinuria and KTDS.
\end{abstract}

Implication for health policy/practice/research/medical education:

In the current study, 35 male Wistar rats were selected. Metabolic syndrome was induced using a fructose-rich diet and then the protective effects eugenol 50 and $100 \mathrm{mg} / \mathrm{kg} / \mathrm{d}$ doses on renal damage due to metabolic syndrome were investigated. The results of this study indicated the improvement of renal functioning and decrease in lipid peroxidation, although eugenol doses used in this study did not reduce proteinuria and kidney tissue damage score.

Please cite this paper as: Kourkinejad Gharaei F, Lakzaei H, Niazi AA, Jahantigh M, Shahraki MR, Safari T. The protective effects of eugenol on metabolic-syndrome, renal damages. J Renal Inj Prev. 2022; 11(1): e04. doi: 10.34172/jrip.2022.04.

\section{Introduction}

Metabolic syndrome includes a group of disorders such as abdominal obesity, hyperglycemia, dyslipidemia and hypertension (1-3). They are associated with insulin resistance, often together with weight gain and obesity. People suffering from metabolic syndrome are at increased risk of diabetes, renal and cardiovascular diseases $(3,4)$. New evidence suggests that the imminence of chronic renal illness is higher in people with metabolic syndrome(5, 6). According to recent studies, prevalence of metabolic syndrome is $34 \%$ globally (7). Important factors in the development of this disorder are high carbohydrate and 
high-fat diets $(8,9)$. Studies have shown that application of fructose as a refined sweetener in modern nutrition is increasing, with daily intake amounting from $16-20 \mathrm{~g}$ to 85-100 g (10,11).

Various studies have suggested different pathways for tissue damage in the metabolic syndrome. One of the pathways is inducing and producing oxidative stress and free radicals respectively. The metabolic syndrome increases free radicals, namely, reactive oxygen species that lead to imbalances in antioxidant activities leading to cellular dysfunctioning $(12,13)$. According to the current studies, oxidative stress plays some important roles in the happening of different diseases including kidney damage (14).

4-allyl-2-methoxyphenol also called as eugenol is a phenolic compound which is utilized being an additive in food production and fragrant (15). Many plants like clove, basil, and nutmeg extracts are saturated with eugenol. Biological activities of eugenol include antioxidant, neuroprotective, anti-inflammatory etc (16). Sheen and colleagues demonstrated eugenol protective effects against oxidative stress and gentamicin-induced nephrotoxicity (2).

\section{Objectives}

Considering the importance of the metabolic syndrome and the protective role of eugenol, the subsequent questions are propounded: 1) Do biochemical parameters' levels in serum and kidney tissue vary after the injection of eugenol in rats affected with metabolic syndrome? 2) Are there any pathological alterations in nephric parenchyma after the application of eugenol in rats with induced metabolic syndrome? Thus this study was designed for assessment of eugenol's role in prevention of renal damages because of metabolic syndrome.

\section{Materials and Methods \\ Animals}

In the current study, 35 Wistar rats which all had male gender and accidentally selected in the weight range of $184.4 \pm 7.2 \mathrm{~g}$ which were prepared from Zahedan University of Medical Sciences' animal center. The environmental temperature of storage place was 23 to $25^{\circ} \mathrm{C}$. Rats had freeform water reaching, standardized rat food nourishment, and 12 hours light as daylight exposure and 12 hours darkness as night time. One week before the test they also adapted to their diet.

\section{Medications}

D-fructose $>99 \%$ was purchased from Syarikat System Malaysia Company. Eugenol was gained from Sigma (St. Louis, MO, USA). Sweet almond oil was procured from Kimiagartoos Pharmaceutical Company in Iran.

\section{Experiment's protocol}

The animals randomly were allocated into five groups including 1) tap water; 2) water with fructose 10\%; 3) water with fructose $10 \%$ plus sweet almond oil and administered intraperitoneally; 4) water with fructose $10 \%$ plus eugenol $50 \mathrm{mg} / \mathrm{kg} / \mathrm{d}$ (17) dissolved in sweet almond oil and administered intraperitoneally; 5) water with fructose $10 \%$ plus eugenol $100 \mathrm{mg} / \mathrm{kg} / \mathrm{d}$ (17) dissolved in sweet almond oil and administered intraperitoneally. The above rats consumed the water saturated with fructose for 60 days(18). After 30 days, on the next day, eugenol and almond oil injection started for duration of 30 days. Throughout the survey, every day the animal's net weight was determined and registered. One day after the administration completion, anesthetizing with the mixture of ketamine ( $75 \mathrm{mg} / \mathrm{kg}$, intraperitoneally) and 10 $\mathrm{mg} / \mathrm{kg}$ intraperitoneally xylazine occurred and then blood samples from the heart of individual animal were taken.

\section{Measurements}

Utilization of Pars Azmoon's kits (from Iranian company) the levels of serum creatinine (Cr), blood urea nitrogen (BUN) and proteinuria were measured quantitatively. We determined nitrite oxide stable metabolite and nitrite level in serum and supernatant via the Griess reaction of colorimetric assay kit (ZellBio, Germany). Homogenized tissue supernatant and serum level of malondialdehyde (MDA) were calculated manually $(19,20)$. Before tissue staining we use $10 \%$ formalin for fixation and paraffin. We colored tissue sections with hematoxylin-eosin staining prior to their examination. Then two pathologists assessed the slides blindly. Tissue pathology was assessed by vehemence of distal tubular damages, transparent renal cast, wastages and debris, flattening and demolition of tubular cell, vacuolization and widening of tubular lumen and scored from 1 to 4 . Overall score was determined as kidney tissue damage score (KTDS) which was reported 1 to 4 , whereas natural tubes without any damage considered zero scores.

\section{Statistical analysis}

By means of mean \pm SEM the data are reported. One-way analysis of variance (ANOVA) was applied in analysis if MDA, nitrite, Cr, BUN laboratory test results and kidney weights. Then the post hoc Tukey test also has been done. Evaluation of histopathological results was conducted with Kruskal-Wallis and Mann-Whitney $U$ tests. We invoked SPSS version $16^{\text {th }}$ for data analysis. $P$ values lower than 0.05 were cogitated significant statistically.

Results

Collation of mean delta weight of animals in different groups showed a significant difference amongst the group receiving fructose besides tap water group $(P<0.05)$. Treatment with eugenol significantly reduces body weight in comparison to fructose ones $(P<0.05)$ as revealed in Figure 1A. It should be noted that the mean left kidney weight in the dissimilar groups did not demonstrate a 
significant difference (Figure 1B).

The results showed that the induction of metabolic syndrome following renal injury has significantly increased serum BUN and Cr levels in fructose in comparison with tap water group $(P<0.001)$. Applying eugenol at dosages of 50 and 100 resulted in a significant reduction in serum BUN and Cr levels in comparison with the fructose group $(P<0.05)$. This two administrations of eugenol (50 and $100 \mathrm{mg} / \mathrm{kg} / \mathrm{d}$ ) have the same effects on BUN and $\mathrm{Cr}$ levels (Figure 1C and D). The mean serum nitrite and MDA levels did not display any significant differences amongst the studied groups (Figure 1A and B). However, the kidney level of MDA and nitrite increased with the induction of metabolic syndrome in the fructose group and treatment with a dose of 50 eugenol decreased MDA level $(P<0.05)$ as shown in Figure 2C and D. On the other hand, the levels of renal nitrite among different groups are not significantly different (Figure 2). The amount of urine protein suggests a kidney damage following the induction of a metabolic syndrome in fructose comparison to the tap water recipient group $(P<0.001)$ as shown in Figure 3. It is noteworthy that treatment with eugenol did not affect the level of this factor with any of the used doses. Tissue damage assessment by KTDS among the groups suggests that the metabolic syndrome caused kidney damage $P<0.01$ ), but treatment with eugenol did not affect the KTDS (Figure 3).

\section{Discussion}

The results of this study indicate renal injury after induction of metabolic syndrome. Despite improvements in renal function, factors such as serum BUN and Cr levels by eugenol, KTDS and proteinuria are not affected by treatment with different eugenol doses.

Several studies approve the antioxidant and antiinflammatory properties of eugenol $(1,16,21)$.Their reports also indicate that eugenol performs as a free radical collector and reduces oxidative stress, lipid peroxidation, and improves antioxidant defense. The results of their studies indicate an enhancement in the glutathione's level, superoxide dismutase, reducing tumor necrosis factor alpha and prostaglandin E2. Eugenol can also reduce MDA by decreasing lipid peroxidation. Thus, the effects of eugenol are the improvement of antioxidant enzymes together with decreasing the inflammatory factors (15).

Another study has reported that eugenol improves the antioxidant capacity and arsenic trioxide -induced nephrotoxicity (21). In this regard, there is evidence that eugenol improved gentamicin nephrotoxicity by reparation oxidative stress, collecting free radicals and diminishing peroxidation of lipid and improving antioxidant defense. In fact, eugenol has its own effects by improving lipid peroxidation, increasing antioxidant defense, eventually improving tissue hypoxia (1).

In this regard, eugenol by its hydroxyphenolic group eliminates electrons from free radicals and prevents oxidation of $\mathrm{Fe}^{2+}$ by $\mathrm{H} 2 \mathrm{O} 2$. Consequently, it reduces radical $\dot{\mathrm{OH}}$ production, which begins with lipid peroxidation. There are also reasons that eugenol reduces cytokines by suppressing cyclooxygenase II and inhibits cell proliferation (1).

Garud et al reported the effects of eugenol on diabetic nephropathy. They showed that eugenol at dosages of 5 and $10 \mathrm{mg} / \mathrm{kg}$ for 28 days reduced the gene-expression
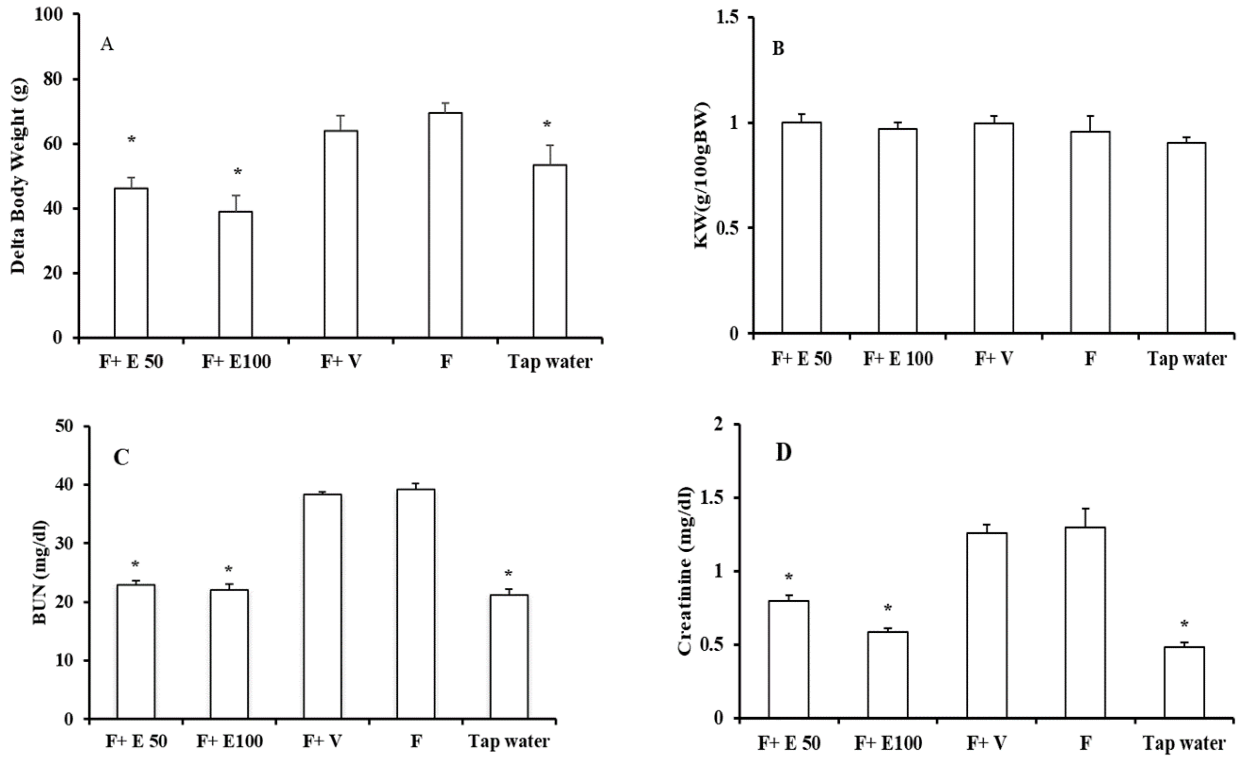

Figure 1. Evaluation of body weight (A),left kidney weight, g/100g BW (B), BUN, serum level of blood urea nitrogen (C) \& creatinine (D). The groups taken tap water, fructose $(F)$, Fructose+ eugenol $50 \mathrm{mg} / \mathrm{kg}(\mathrm{F}+\mathrm{E} 50)$, Fructose+ eugenol $100 \mathrm{mg} / \mathrm{kg}(\mathrm{F}+\mathrm{E} 100)$ and $\mathrm{Fructose}+$ almond oil $(\mathrm{F}+\mathrm{V})$. ${ }^{*}$ Significant differences compared to fructose and fructose+ almond oil group $(P$ value $<0.05)$. 

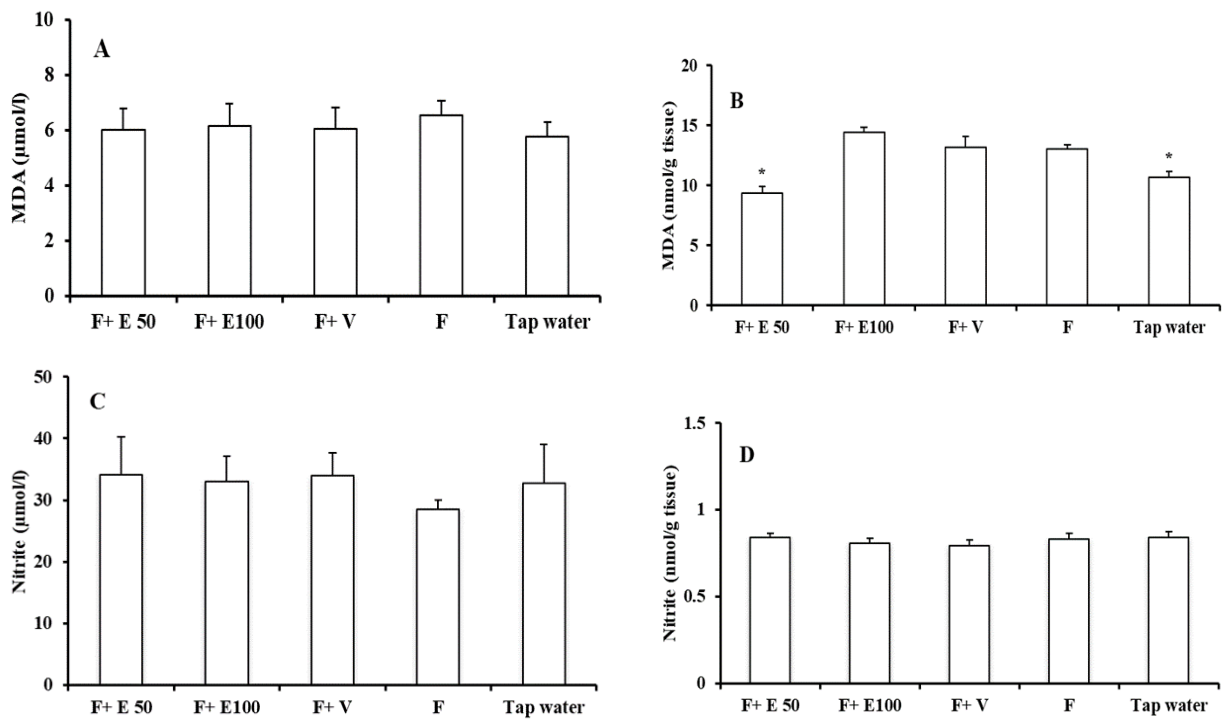

Figure 2. Evaluation of MDA`s serum level (A), kidney level of MDA (B), serum level of nitrite (C) and kidney level of nitrite (D). The groups received, tap water, fructose $(F)$, Fructose+ eugenol $50 \mathrm{mg} / \mathrm{kg}(\mathrm{F}+\mathrm{E} 50)$, Fructose+ eugenol $100 \mathrm{mg} / \mathrm{kg}(\mathrm{F}+\mathrm{E} 100)$ and Fructose+ almond oil $(\mathrm{F}+\mathrm{V})$. * Significant differences compared to fructose and fructose+ almond oil group $(P<0.05)$.
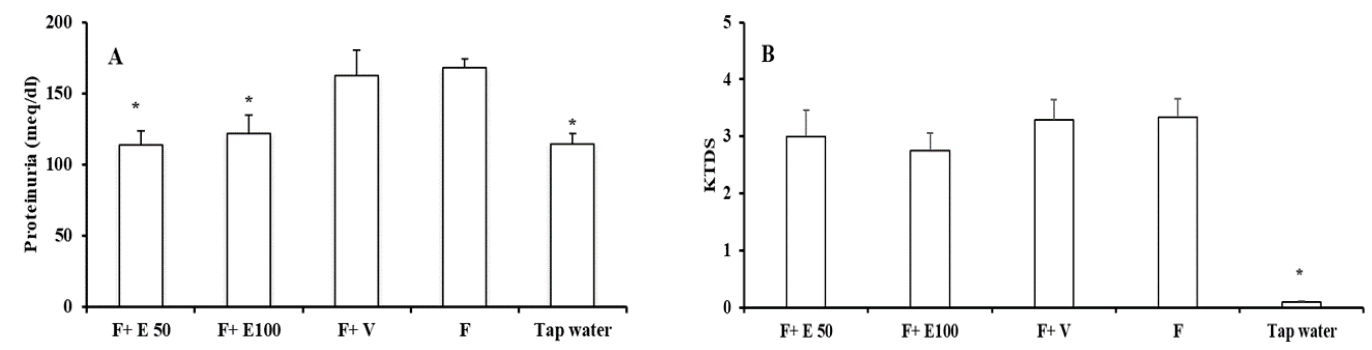

C

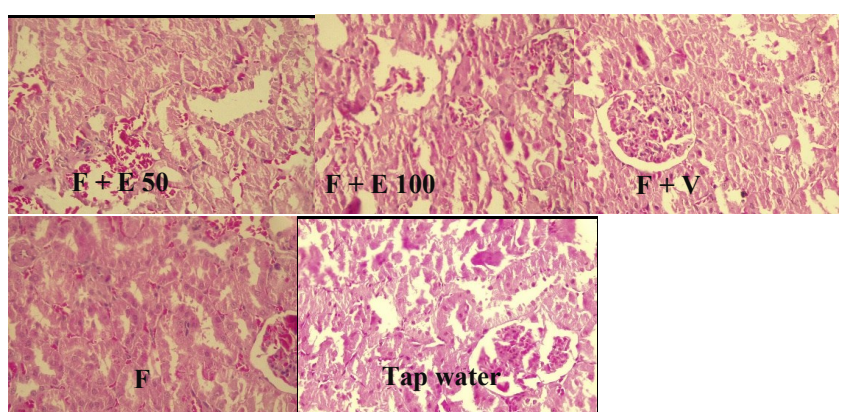

Figure 3. Evaluation of urine protein (A), KTDS, kidney tissue damage score (B) and pathology images (magnification $\times 400$ ) of kidney tissue (C). The groups received, tap water, fructose (F), Fructose+ eugenol $50 \mathrm{mg} / \mathrm{kg}(\mathrm{F}+\mathrm{E} 50)$, Fructose+ eugenol $100 \mathrm{mg} / \mathrm{kg}(\mathrm{F}+\mathrm{E} 100)$ and Fructose+ almond oil $(\mathrm{F}+\mathrm{V})$. * Significant differences compared to fructose and fructose+ almond oil group $(P<0.05)$.

of transforming growth factor beta 1 (TGF- $\beta$ 1) level and improved tissue damage in diabetic rats (22).

\section{Conclusion}

Our results also indicate improved BUN, Cr and decrease in lipid peroxidation, although eugenol doses used in this study did not reduce proteinuria and KTDS. This finding may be related to the applied doses or their duration.
Authors' contribution

TS and FK planned, guided, oversaw and evaluated and analyzed the study and ready the first draft of document. HL, FK, AN, MJ, MS and TS partaken in the running of the study and gathered the data. TS and FK shared in the inscription and correcting of the article. All authors participated in preparing the final draft of the manuscript, revised the manuscript and critically evaluated the 
intellectual contents. All authors have read and approved the content of the manuscript and confirmed the accuracy or integrity of any part of the work.

\section{Conflicts of interest}

The authors declare that they have no competing interests.

\section{Ethical issues}

This study was confirmed by the Ethics Committee of Zahedan University of Medical Sciences. As an experiment, the protocols were approved to be in accordance with the guidelines of Animal Ethics Committee of Zahedan University of Medical Sciences, Zahedan, Iran (IR. ZAUMS.REC.1397.258). This study was extracted from the MD thesis of Fatemeh Kourkinejad at this University (Thesis \#1397-8997). Moreover, ethical issues (including plagiarism, data fabrication, double publication) have been completely observed by the authors.

\section{Funding/Support}

The research was corroborated by Zahedan University of Medical Sciences (Grant \#8997).

\section{References}

1. Said MM. The protective effect of eugenol against gentamicin-induced nephrotoxicity and oxidative damage in rat kidney. Fundam Clin Pharmacol. 2011;25(6):708-16, doi: $\quad 10.1111 / j .1472-8206.2010 .00900 . x$.

2. Sheen Y-J, Sheu WH-H. Metabolic syndrome and renal injury. Cardiol Res Pract. 2011;2011:567389 doi: 10.4061/2011/567389

3. Pei D, Kuo SW, Wu DA, Lin TY, Hseih MC, Lee CH, et al. The relationships between insulin resistance and components of metabolic syndrome in Taiwanese Asians. Int J Clin Pract. 2005;59:1408-16. doi: 10.1111/j.1368-5031.2005.00661.x

4. Laguardia HA, Hamm LL, Chen J. The metabolic syndrome and risk of chronic kidney disease: Pathophysiology and intervention strategies. J Nutr Metab. 2012;2012: 652608 doi: $10.1155 / 2012 / 652608$

5. Lee I-T, Huang C-N, Lee W-J, Lee H-S, Sheu WH-H. Aggravation of albuminuria by metabolic syndrome in type 2 diabetic Asian subjects. Diabetes Res Clin Pract. 2008;81:345-50. doi: 10.1016/j.diabres.2008.05.010

6. Chen J, Muntner P, Hamm LL, Jones DW. The metabolic syndrome and chronic kidney disease in US adults. Ann Intern Med. 2004;140:167. doi: 10.7326/0003-4819-140-3200402030-00007

7. Eckel RH, Grundy SM, Zimmet PZ. The metabolic syndrome. Lancet. 2005;365:1415-28. doi: 10.1016/S01406736(05)66378-7

8. Panchal SK, Brown L. Rodent models for metabolic syndrome research. J Biomed Biotechnol. 2011;2011:351982.

\section{doi: $\quad 10.1155 / 2011 / 351982$}

9. Moreno-Fernández S, Garcés-Rimón M, Vera G, Astier J, Landrier J, Miguel M. High Fat/High Glucose Diet Induces Metabolic Syndrome in an Experimental Rat Model. Nutrients. 2018;10:1502. doi: 10.3390/nu10101502

10. Tappy L, Lê K-A. Metabolic effects of fructose and the worldwide increase in obesity. Physiol Rev. $2010 ; 90(1): 23$ 46. doi: 10.1152/physrev.00019.2009

11. Bray GA, Nielsen SJ, Popkin BM. Consumption of highfructose corn syrup in beverages may play a role in the epidemic of obesity. Am J Clin Nutr. 2004;79(4):537-43. doi: 10.1093/ajen/79.4.537

12. Ceriello A, Motz E. Is oxidative stress the pathogenic mechanism underlying insulin resistance, diabetes, and cardiovascular disease? The common soil hypothesis revisited. Arterioscler Thromb Vasc Biol. 2004;24:816-23. doi: $\quad$ 10.1161/01.ATV.0000122852.22604.78

13. Roberts CK, Sindhu KK. Oxidative stress and metabolic syndrome. Life Sci. 2009;22;84:705-12. doi: 10.1016/j. lfs.2009.02.026

14. Wan C, Su H, Zhang C. Role of NADPH oxidase in metabolic disease-related renal injury: an update. Oxid Med Cell Longev. 2016;2016:7813072. doi: 10.1155/2016/7813072

15. Barhoma RA. The role of eugenol in the prevention of chromium-induced acute kidney injury in male albino rats. Alexandria Med J. 2018;54(4):711-5.

16. Pramod K, Ansari SH, Ali J. Eugenol: a natural compound with versatile pharmacological actions. Nat Prod Commun. 2010;5:1999-2006.

17. Daniel AN, Sartoretto SM, Schmidt G, Caparroz-Assef SM, Bersani-Amado CA, Cuman RKN. Anti-inflammatory and antinociceptive activities $\mathrm{A}$ of eugenol essential oil in experimental animal models. Revista Brasileira de Farmacognosia. 2009;19:212-7.

18. Bantle JP. Dietary fructose and metabolic syndrome and diabetes. J Nutr. 2009;139:1263S-8S. doi: 10.3945/ jn.108.098020

19. El-Beshbishy HA, Bahashwan SA, Aly HA, Fakher HA. Abrogation of cisplatin-induced nephrotoxicity in mice by alpha lipoic acid through ameliorating oxidative stress and enhancing gene expression of antioxidant enzymes. Eur J Pharmacol. 2011;668:278-84. doi: 10.1016/j. ejphar.2011.06.051

20. Tsikas D. Analysis of nitrite and nitrate in biological fluids by assays based on the Griess reaction: appraisal of the Griess reaction in the L-arginine/nitric oxide area of research. J Chromatogr B Analyt Technol Biomed Life Sci. 2007;15;851:51-70.

21. Pace C, Dagda R, Angermann J. Antioxidants protect against arsenic induced mitochondrial cardio-toxicity. Toxics. 2017;5:E38. doi: 10.3390/toxics5040038

22. Garud MS, Kulkarni YA. Eugenol ameliorates renal damage in streptozotocin-induced diabetic rats. Flavour Fragr J. 2017;32:54-62.

Copyright (c) 2022 The Author(s); Published by Nickan Research Institute. This is an open-access article distributed under the terms of the Creative Commons Attribution License (http://creativecommons.org/licenses/by/4.0), which permits unrestricted use, distribution, and reproduction in any medium, provided the original work is properly cited. 